\title{
INVESTIGACIONES
}

\section{Talento académico, vulnerabilidad escolar y resultados en la prueba de selección universitaria*}

\author{
Giftedness, school vulnerability and university admission test scores
}

\author{
Pablo González Adonis, ${ }^{a}$ Violeta Arancibia Clavel, ${ }^{a b}$ Diana Boyanova ${ }^{c}$ \\ aPontificia Universidad Católica de Chile \\ Telf.: (56) 223547635. Correo electrónico: psgonzal@uc.cl \\ 'Telf.: (56) 992209532. Correo electrónico: varancib@mac.com \\ cPrometriks (Senior Psychometrician), Bulgaria \\ Correo electrónico: dboyanova@prometriks.com
}

\begin{abstract}
RESUMEN
En la presente investigación se analizó la relación existente entre el potencial de talento académico, las habilidades cognitivas, la participación en un programa de talentos, la vulnerabilidad escolar y los resultados en la prueba de selección universitaria (PSU) en 1303 estudiantes que postularon en $5^{\circ}$ básico al Programa PentaUC, entre los años 2001 y 2004, y que rindieron la PSU entre los años 2007 y 2010. A nivel general, los resultados muestran que los estudiantes que finalizan un programa de talentos obtienen mejores resultados PSU que quienes no lo terminan. La vulnerabilidad de los contextos académicos y el tipo de contenidos evaluados en la PSU aparecieron como variables que modulan tanto el efecto del Programa como el efecto del potencial de talento sobre los puntajes PSU. Las altas habilidades cognitivas no parecen tener influencia directa sobre los puntajes PSU cuando estos se encuentran en el extremo superior de la escala.
\end{abstract}

Palabras clave: PSU, habilidades cognitivas, PentaUC.

\begin{abstract}
Relation between giftedness, cognitive abilities, participation in a gifted program, school vulnerability and the Chilean university admission test scores was studied in a sample of 1303 students who applied to PentaUC Program between 2001 and 2004, and took the Chilean University Admission Test (PSU) between 2007 and 2010. Results show that students who complete a gifted program obtain better PSU scores than those who drop out. Vulnerability of academic contexts and the type of content evaluated in PSU, appeared as variables that modulate both the effect of the program and the effect of giftedness on PSU scores. High cognitive abilities seem to have no direct influence on PSU scores when those scores are at the upper end of the scale.
\end{abstract}

Key words: PSU, cognitive abilities, PentaUC.

La investigación fue financiada por el Fondo Nacional de Desarrollo Científico y Tecnológico (FONDECYT), proyecto Fondecyt Regular 2011, $\mathrm{n}^{\circ} 1110948$. 


\section{DESARROLLO DEL TALENTO ACADÉMICO}

El talento académico es definido como un desempeño destacado en algún área académica o científica del quehacer humano, y que implica ubicarse en el 10\% superior en comparación al desempeño logrado por el grupo de pares (Gagné, 2004). El talento académico requiere de un proceso de aprendizaje y práctica formal e informal para poder desarrollarse, y son las habilidades cognitivas elevadas signos de potencial talento académico. La presencia de estos precursores no aseguran, sin embargo, desempeños destacados en el futuro (Gagné, 2004; Renzulli, 2012).

En los estudiantes con potencial de talento, el desarrollo final del mismo depende de factores intrapersonales tales como la motivación, la voluntad, el auto concepto, la auto regulación y las creencias de auto eficacia, así como de factores ambientales, como por ejemplo expectativas familiares y programas de enriquecimiento académico, todos los cuales actúan como catalizadores, facilitando o inhibiendo la expresión del potencial y su desarrollo (Gagné, 2004; Renzulli, 2012).

Cuando los catalizadores no son adecuados, la probabilidad de no desarrollar el talento académico aumenta. Por ejemplo, la falta de desafíos académicos óptimos, acordes a las habilidades de individuos con potencial de talento, puede generar desmotivación, pérdida de interés en los estudios y, consecuentemente, malos resultados académicos (Arancibia, 2009; Freeman, 2005; Plank \& Jordan, 2001). Por otra parte, estudiantes en entornos vulnerables y de bajos recursos económicos están también expuestos a no desarrollar el talento, y no solamente por la falta de recursos, sino también porque sus entornos familiares y comunitarios no construyen altas expectativas respecto de su posibilidad de alcanzar estudios superiores y logros académicos (Coleman, 1988; Plank \& Jordan, 2001). Cuando muchas de estas características se presentan simultáneamente, las oportunidades para que los estudiantes con potencial de talento tengan acceso a los catalizadores ambientales adecuados disminuyen (Gagné, 2004).

En el caso de Chile, el foco que el sistema educacional pone en el desarrollo académico del estudiante promedio (Benavides, Ríos \& Marshal, 2004) contribuye a que la pérdida del talento académico sea una situación probable, especialmente en los contextos vulnerables, ya que el foco en el estudiante promedio descuida tanto a aquellos alumnos con dificultades académicas como a aquellos con una velocidad de aprendizaje alta. Debido a esto, el año 2001 se creó el Programa para niños y jóvenes con talento académico de la Pontificia Universidad Católica de Chile (PentaUC), cuyo propósito fue actuar como un catalizador para el desarrollo del talento académico en estudiantes de contextos económicamente vulnerables.

PentaUC es un programa de enriquecimiento académico, extra curricular, en donde el estudiante puede elegir 2 cursos y 1 taller por semestre, y permanecer como alumno regular hasta un máximo de 7 años. Los estudiantes acceden al proceso de postulación una vez que son nominados por sus colegios o sus padres. Cada uno de los postulantes es evaluado con el Test de Matrices Progresivas de Raven, escala general, siendo seleccionados aquellos cuyos resultados se encuentran en el percentil 75 de la muestra de postulantes ${ }^{1}$.

Considerando los antecedentes teóricos señalados y el proceso de selección de PentaUC, en el presente artículo se usarán indistintamente las expresiones "estudiantes con altas habilidades cognitivas" y "estudiantes con potencial talento académico". 
Siguiendo el modelo de Gagné (2004), el Programa PentaUC busca desarrollar tanto las habilidades cognitivas de sus estudiantes, mediante un currículum especialmente diseñado, como las características socioafectivas que actúan como catalizadores intrapersonales para la regulación, manifestación y desarrollo del talento académico (Arancibia \& González, 2007).

\section{TALENTO ACADÉMICO Y PRUEBAS DE ADMISIÓN A LA EDUCACIÓN SUPERIOR}

En Chile, la Prueba de Selección Universitaria (PSU) fue aplicada por primera vez en 2003 como reemplazo a la Prueba de Aptitud Académica (PAA). La PSU fue implementada en un esfuerzo por corregir las diferencias socioeconómicas que generaba su antecesora. Por ello es una prueba basada en los contenidos del currículum nacional obligatorio, que es enseñado en todos los colegios, independientemente de la dependencia del establecimiento educativo (municipal, particular subvencionada o particular pagada) (Bravo et al., 2008).

La PSU incluye dos pruebas obligatorias (Lenguaje y Matemáticas) y dos opcionales (Historia y Ciencias). Cada una consiste en 75 a 80 preguntas de selección múltiple con cinco alternativas. El puntaje bruto es calculado a partir de la suma de las respuestas correctas, menos un cuarto de la suma de las respuestas incorrectas ${ }^{2}$. Este puntaje es ajustado a una curva normal con media de 500 puntos y desviación estándar de 110 , un mínimo de 150 y un máximo de 850 puntos. Las universidades escogen a los estudiantes a través de los puntajes que obtienen en la PSU y las notas de enseñanza media (NEM, que son ajustadas a la misma escala que la PSU), las cuales son ponderadas según los requerimientos de cada carrera ${ }^{3}$.

En la actualidad la PSU no ha sido capaz de corregir las diferencias socioeconómicas, ya que los estudiantes de mayores recursos tienen acceso a mayores oportunidades de preparación, y debido a que la proporción del currículum escolar que es cubierto efectivamente está altamente correlacionado con el nivel socioeconómico del colegio (Beyer, 2009; Koljatic \& Silva, 2010; OECD-World Bank, 2009; Pearson, 2013). Los colegios con mayor índice de vulnerabilidad suelen cubrir una proporción menor de los contenidos curriculares (Pearson, 2013) debido, entre otras causas, a poseer profesores con menor calificación (Meckes \& Bascopé, 2009; Ortúzar et al., 2009) y con poca flexibilidad (Paredes \& Paredes, 2009), así como a la falta de eficiencia de las administraciones de dichos establecimientos (Arancibia, 1992; Paredes \& Paredes, 2009). Al mismo tiempo, son instituciones que deben lidiar con una amplia gama e intensidad de temáticas psicosociales que obligan a utilizar recursos profesionales en su manejo, contribuyendo a un eventual desmedro de los procesos de enseñanza-aprendizaje.

En Chile no hay estudios sobre el desempeño en la PSU de los estudiantes con potencial de talento académico, en términos de la relación entre habilidades cognitivas y puntajes PSU. Sin embargo, hay dos reportes - PentaUC en Santiago (Centro de Políticas Públicas UC, 2009) y DELTA-UCN en Antofagasta (González \& Glavic, 2011)_ que muestran que los estudiantes con potencial talento académico que participan en programas educacionales

A partir del año 2015 la fórmula de cálculo del puntaje bruto dejó de incluir el descuento por respuestas incorrectas.

Desde el año 2012 se incorporó el Ranking de Notas como factor a ponderar en la selección universitaria chilena. Dicho factor no está incluido en este estudio debido a que se utilizaron los resultados PSU entre los años 2007 y 2010. 
especialmente diseñados y que trabajan con estudiantes de bajo nivel socioeconómico, tienen mejor desempeño en la PSU que los estudiantes de nivel socioeconómico similar.

En Estados Unidos hay dos pruebas estandarizadas de admisión a la universidad: el test de razonamiento $\mathrm{SAT}^{4}$ y el American College Testing (ACT). El propósito de la prueba SAT es evaluar habilidades cognitivas tales como razonamiento crítico, las que se suponen son ejercitadas en la escuela. Esta prueba también permite medir inteligencia general y funcionamiento cognitivo (Beaujean et al., 2006; Frey \& Detterman 2004). Por su parte, la prueba ACT tiene como propósito medir habilidades específicas requeridas para alcanzar un buen rendimiento en la universidad (ACT, 2007).

La principal diferencia entre las pruebas SAT y ACT es que la primera tiene un énfasis en la medición de aptitudes cognitivas, mientras que la segunda está orientada a medir cuánto del currículum escolar ha sido aprendido. Por lo tanto, respecto de lo que pretenden medir, la prueba ACT es más parecida a la PSU chilena que la prueba SAT.

En relación al proceso de construcción de estos instrumentos, las tres pruebas mencionadas son piloteadas bajo los supuestos de la Teoría Clásica de Medición (CTT) y también son evaluadas por expertos con el propósito de validarlas y prevenir sesgos (ACT, 2007; DEMRE, 2005; Wolkowitz, 2008). La Teoría Clásica de Medición emplea la dificultad de los ítems (proporción de preguntas correctas para cada ítem) y la discriminabilidad (correlación entre el puntaje de un ítem y el puntaje total) como los parámetros principales para evaluar si las pruebas miden correctamente las habilidades cognitivas y contenidos que pretenden y si distinguen entre los estudiantes con distintos niveles de desarrollo de estas habilidades y logro de aprendizajes. Esta teoría es usada porque es fácilmente aplicable a grupos de individuos grandes y heterogéneos y permite medir conceptos multidimensionales (Dussaillant, 2003).

Brody y Benbow (1990) han mostrado que los programas educativos especiales para estudiantes con potencial talento académico son un factor importante para explicar la diferencia de desempeño entre aquellos que rinden al nivel de sus capacidades y aquellos que rinden bajo su potencial capacidad en la prueba SAT. Más aún, los estudiantes con potencial talento académico que participan de experiencias de enriquecimiento educativo en la infancia y/o adolescencia pueden presentar un mejor desempeño en el futuro, tanto en las pruebas de admisión a la universidad como en la educación superior (Freeman, 2005). Por el contrario, el resultado de la falta de oportunidades es que los niños y adolescentes con potencial de talento rindan en un nivel inferior al que potencialmente podrían haberlo hecho en las pruebas de selección a la educación superior (Plank \& Jordan, 2001).

La literatura ha reportado correlaciones entre el test de Matrices Progresivas de Raven, escala avanzada, y los puntajes en la prueba SAT en un rango entre 0,39 y 0,72 (Frey \& Detterman, 2004; Rhode \& Thompson, 2007). Koenig, Frey y Detterman (2008) señalan una relación significativa entre mediciones de habilidades cognitivas y puntajes de la prueba ACT, presentando correlaciones de 0,61 entre el test de Matrices Progresivas de Raven, escala avanzada, y los puntajes totales de la prueba SAT. A pesar de esta alta correlación, los alumnos con altas habilidades cognitivas, especialmente los de bajo nivel socioeconómico, podrían ser susceptibles de rendir menos de lo que su potencial les permite, porque estos test de admisión están usualmente basados en el currículum más que en las habilidades cognitivas (Zwick, 2002).

\footnotetext{
El acrónimo SAT solía significar "Scholastic Assessment Test". Actualmente dicho nombre no es utilizado, sin embargo, debido a que la mayoría de la gente conocía el instrumento por sus siglas, estas se mantuvieron.
} 
A pesar de que las medidas de habilidad cognitiva general muestran correlaciones significativas con el logro académico, incluido el desempeño en las pruebas de admisión, la habilidad cognitiva general, por sí misma, no explica más del 50\% de la varianza asociada con el logro académico (Jensen, 1998, Cit. en Rhode \& Thompson, 2007). Variables diferentes a la habilidad cognitiva general, por ejemplo ciertas habilidades cognitivas específicas, pueden ser más influyentes en el logro académico (Rhode \& Thompson, 2007).

\section{OBJETIVOS DEL ESTUDIO}

En un contexto educativo desigual (como el chileno) es fundamental desarrollar conocimiento que permita implementar mejoras en los procesos de aprendizaje de todos los estudiantes. Al mismo tiempo, en la medida en que la PSU siga siendo el principal mecanismo de admisión a la educación superior, es pertinente explorar las variables que favorecen obtener buenos resultados, sobre todo en estudiantes que enfrentan la evaluación con desventajas debidas a sus contextos académicos de origen.

En atención a lo mencionado, el presente estudio pretende indagar en la relación existente entre el potencial de talento académico, la participación en un programa de desarrollo del talento académico, las habilidades cognitivas, la vulnerabilidad escolar y los resultados en la PSU y en el colegio, en una muestra de estudiantes chilenos.

Para ello se busca comparar los resultados PSU, y en el liceo, de estudiantes identificados (o no) como con potencial talento académico, y evaluar las diferencias en dichos resultados en función de la vulnerabilidad escolar de los estudiantes y en base a su nivel de participación en un programa de desarrollo del talento académico.

Además, se quiere explorar el impacto de las notas de enseñanza media, la vulnerabilidad escolar y las habilidades cognitivas sobre los puntajes PSU de estudiantes que participaron (o no) en un programa de desarrollo del talento académico.

\section{METODOLOGÍA}

\subsection{PARTICIPANTES}

Los participantes son 1303 estudiantes que postularon en $5^{\circ}$ básico al Programa PentaUC entre los años 2001 y 2004, y que rindieron la PSU entre los años 2007 y 2010. El total de participantes fue dividido en 3 grupos: i) grupo No Seleccionados, compuesto por estudiantes que postularon, pero que no obtuvieron el puntaje mínimo en el test de Raven para ser aceptados en el Programa, ii) grupo Retirados, compuesto por estudiantes aceptados en el Programa, pero que no lo finalizaron, y iii) grupo Graduados, integrado por estudiantes seleccionados que completaron el ciclo completo del Programa, desde $6^{\circ}$ básico hasta $4^{\circ}$ medio.

De la muestra total el 58,6\% son hombres. En la Tabla 1 se presenta cada grupo y su constitución por sexo ${ }^{5}$.

Para efectos de los análisis de este estudio, la variable sexo no fue considerada, debido a que su inclusión hubiese contribuido a crear grupos con cantidades muy pequeñas de casos. 
Estudios Pedagógicos XLIII, N $1:$ 171-191, 2017

TALENTO ACADÉMICO, VULNERABILIDAD ESCOLAR Y RESULTADOS EN LA PRUEBA DE SELECCIÓN UNIVERSITARIA

Tabla 1. Composición de la muestra según sexo

\begin{tabular}{|l|c|c|c|}
\hline \multirow{2}{*}{ Participación en PentaUC } & \multicolumn{2}{|c|}{ Sexo } & \multirow{2}{*}{ Total } \\
\cline { 2 - 3 } & Hombres & Mujeres & \\
\hline No Seleccionados & 594 & 429 & 1023 \\
\hline Retirados & 131 & 76 & 207 \\
\hline Graduados & 38 & 35 & 73 \\
\hline & 763 & 540 & 1303 \\
\hline
\end{tabular}

\subsection{VARIABLES}

Participación en el Programa PentaUC y Potencial Talento Académico: Se consideraron, como fue señalado, tres grupos: No Seleccionados, Retirados y Graduados. Los dos últimos corresponden a estudiantes identificados como estudiantes con potencial talento académico según sus resultados en el proceso de selección a PentaUC.

Habilidades Cognitivas: Se utilizó el Test de Matrices Progresivas de Raven, escala general (Raven, Court \& Raven, 1996), como medida de la inteligencia general y habilidades analíticas.

Vulnerabilidad Escolar: Se utilizó el índice de vulnerabilidad escolar (IVE) asociado al colegio de procedencia del estudiante al momento de postular a PentaUC. Este indicador, construido por La Junta Nacional de Auxilio Escolar y Becas de Chile (JUNAEB), señala el porcentaje de alumnos que se encuentran en situación de vulnerabilidad en cada escuela. La JUNAEB define la vulnerabilidad como una:

condición dinámica que resulta de la interacción de una multiplicidad de factores de riesgo y protectores, que ocurren en el ciclo vital de un sujeto y que se manifiestan en conductas o hechos de mayor o menor riesgo social, económico, psicológico, cultural, ambiental y/o biológico, produciendo una desventaja comparativa entre sujetos, familias y/o comunidades. (Cornejo et al., 2005, p. 14).

Para efectos de este estudio se utilizó el IVE 2004 para escuelas básicas, que es el índice más antiguo publicado en las bases de datos disponibles al momento de la consolidación de datos (JUNAEB, 2012). Al analizar la información disponible en JUNAEB se constata que los IVE de los establecimientos no registran mayores variaciones de un año a otro, es por esto que se decidió utilizar el IVE 2004 y aplicarlo a todos los sujetos de la muestra, independientemente de su año de postulación.

Rendimiento en test de admisión a la educación superior: Para este efecto se utilizaron los Puntajes PSU de los participantes. Los datos fueron obtenidos a través de la información pública que todos los años da a conocer el Departamento de Evaluación, Medición y Registro Educacional de la Universidad de Chile (DEMRE) ${ }^{6}$. Se registraron los resultados para las PSU Lenguaje, Matemática, Historia y Ciencias.

\footnotetext{
6 Organismo encargado de la administración del proceso de selección y admisión a la educación superior de las universidades del Consejo de Rectores (universidades tradicionales) y las universidades privadas adscritas.
} 
Rendimiento escolar: Acá fue utilizado el puntaje NEM, asociado a las calificaciones durante la educación media. Los datos también fueron obtenidos a través de la información pública que da a conocer DEMRE. Este indicador fue considerado como una variable representativa tanto de la adquisición de conocimientos enseñados en el colegio como del nivel en el que los estudiantes presentarían características propias para el rendimiento académico, tales como constancia, capacidad de organización, capacidad para el estudio, responsabilidad, entre otros.

\subsection{PROCEDIMIENTO}

Una vez recolectados los datos, se procedió a establecer la organización en los tres grupos de Participación en PentaUC (No Seleccionados, Retirados y Graduados).

Los IVE fueron estandarizados para la muestra total y, a partir de este procedimiento, se construyeron 3 niveles de vulnerabilidad (Baja, Media y Alta) con aproximadamente un tercio de los participantes cada uno.

Mezclando las dos clasificaciones, quedaron definidos 9 grupos (ver Tabla 2).

Tabla 2. Composición de la muestra según vulnerabilidad y participación en PentaUC

\begin{tabular}{|l|c|c|c|c|}
\hline \multirow{2}{*}{ Participación en PentaUC } & \multicolumn{3}{|c|}{ Nivel de Vulnerabilidad } & \multirow{2}{*}{ Total } \\
\cline { 2 - 4 } & Baja & Media & Alta & \\
\hline No Seleccionados & 291 & 348 & 384 & 1023 \\
\hline Retirados & 104 & 57 & 46 & 207 \\
\hline Graduados & 34 & 26 & 13 & 73 \\
\hline & 429 & 431 & 443 & $1303^{7}$ \\
\hline
\end{tabular}

Para la comparación de los resultados PSU entre los grupos se calcularon las medias de cada uno y se realizaron pruebas ANOVA de un factor. Se llevaron a cabo pruebas post hoc para determinar el detalle de las diferencias significativas, cuando estas aparecieron.

Finalmente, se ejecutaron análisis de regresión simple con cada PSU como variable dependiente, en cada uno de los tres grupos de participación en PentaUC. Para efectos de estos análisis no se consideraron los grupos de vulnerabilidad, puesto que el IVE se utilizó como variable independiente de los modelos predictivos a evaluar.

Los siguientes análisis pueden mostrar diferencias respecto del número total de participantes señalados. Esto se debe a que no toda la información en cuanto a puntajes PSU, IVE o NEM estuvo disponible para el 100\% de participantes. 
Estudios Pedagógicos XLIII, N ${ }^{\circ}$ : 171-191, 2017

TALENTO ACADÉMICO, VULNERABILIDAD ESCOLAR Y RESULTADOS EN LA PRUEBA DE SELECCIÓN

UNIVERSITARIA

\section{RESULTADOS}

Las tablas 3 y 4 muestran los resultados PSU de cada grupo. Se realizaron pruebas ANOVA para comparar los promedios en cada una de las PSU; todos mostraron diferencias significativas entre los grupos (PSU Lenguaje: $\mathrm{F}(8,995)=30.29, \mathrm{p} \leq .001$; PSU Matemáticas: $\mathrm{F}(8,995)=38.21, \mathrm{p} \leq .001$; PSU Historia: $\mathrm{F}(8,593)=12.17, \mathrm{p} \leq .001$; PSU Ciencias: $\mathrm{F}(8$, $556)=20.25, \mathrm{p} \leq .001 ; \mathrm{NEM}: \mathrm{F}(8,1057)=7.566, \mathrm{p} \leq .001)$.

Tabla 3. Resultados PSU Lenguaje y Matemáticas según Vulnerabilidad y Participación en PentaUC

\begin{tabular}{|l|c|c|c|c|c|}
\hline \multirow{2}{*}{ Participación en PentaUC } & \multirow{2}{*}{ Vulnerabilidad } & \multicolumn{2}{|c|}{ PSU Lenguaje } & \multicolumn{2}{c|}{ PSU Matemáticas } \\
\cline { 3 - 6 } & & Promedio & $\mathrm{n}$ & Promedio & $\mathrm{n}$ \\
\hline \multirow{3}{*}{ No Seleccionados } & Baja & 569.76 & 259 & 559.06 & 260 \\
\cline { 2 - 6 } & Media & 520.11 & 252 & 508.63 & 251 \\
\cline { 2 - 6 } & Alta & 498.37 & 257 & 480.1 & 258 \\
\hline \multirow{3}{*}{ Retirados } & Baja & 596.74 & 92 & 594.33 & 91 \\
\cline { 2 - 6 } & Media & 568.5 & 42 & 569.26 & 42 \\
\cline { 2 - 6 } & Alta & 562.39 & 31 & 561.35 & 31 \\
\hline \multirow{3}{*}{ Graduados } & Baja & 646.85 & 34 & 663.56 & 34 \\
\cline { 2 - 6 } & Media & 638.5 & 24 & 632.79 & 24 \\
\cline { 2 - 6 } & Alta & 589.15 & 13 & 578.23 & 13 \\
\hline
\end{tabular}

Tabla 4. Resultados PSU Historia, PSU Ciencias y Puntaje NEM, según Vulnerabilidad y Participación en PentaUC

\begin{tabular}{|l|c|c|c|c|c|c|c|}
\hline \multirow{2}{*}{ Participación en PentaUC } & \multirow{2}{*}{ Vulnerabilidad } & \multicolumn{2}{|c|}{ PSU Historia } & \multicolumn{2}{|c|}{ PSU Ciencias } & \multicolumn{3}{c|}{ NEM } \\
\cline { 3 - 9 } & & Promedio & $\mathrm{n}$ & Promedio & $\mathrm{n}$ & Promedio & $\mathrm{n}$ \\
\hline \multirow{4}{*}{ No Seleccionados } & Baja & 574.63 & 144 & 557.46 & 164 & 573.31 & 264 \\
\cline { 2 - 9 } & Media & 533.39 & 149 & 513.96 & 141 & 560.75 & 273 \\
\cline { 2 - 9 } & Alta & 507.23 & 176 & 492.35 & 109 & 556.9 & 289 \\
\hline \multirow{3}{*}{ Retirados } & Baja & 581.04 & 50 & 618.09 & 54 & 598.8 & 92 \\
\cline { 2 - 9 } & Media & 550.04 & 26 & 555.04 & 25 & 588.33 & 43 \\
\cline { 2 - 9 } Graduados & Alta & 560.87 & 15 & 537.62 & 21 & 585.41 & 34 \\
\cline { 2 - 8 } & Baja & 655.82 & 17 & 664.33 & 24 & 636.38 & 34 \\
\cline { 2 - 8 } & Media & 617.19 & 16 & 612.95 & 19 & 665.32 & 25 \\
\cline { 2 - 8 } & Alta & 573.67 & 9 & 532.13 & 8 & 616.67 & 12 \\
\hline
\end{tabular}


Para determinar dónde se encontraban las diferencias específicas se utilizó la prueba post hoc de Bonferroni para la PSU Matemáticas, PSU Historia, PSU Ciencias y el puntaje NEM, y la prueba C de Dunnet para el caso de la PSU Lenguaje, en donde las varianzas de los grupos resultaron ser no homogéneas (estadístico de Levene para PSU Lenguaje = $0.67, \mathrm{p}=0.031$ ).

\subsection{COMPARACIONES SEGÚN NIVEL DE VULNERABILIDAD}

Para cada una de las pruebas de selección universitaria se compararon los grupos según vulnerabilidad (Baja, Media, Alta) dentro de cada nivel de participación en el Programa (No Seleccionados, Retirados, Graduados) (ver Tabla 5). La intención fue determinar cómo el factor vulnerabilidad incidía en los resultados PSU de los No Seleccionados, Retirados y Graduados del Programa.

Tabla 5. Pruebas Post Hoc según vulnerabilidad, dentro de cada nivel de participación

\begin{tabular}{|c|c|c|c|c|c|}
\hline PSU & Prueba Post Hoc & Grupo (I) & Grupo (J) & $\begin{array}{c}\text { Diferencia de } \\
\text { medias (I-J) }\end{array}$ & $\begin{array}{c}\text { Nivel de } \\
\text { significancia }\end{array}$ \\
\hline \multirow[t]{9}{*}{ Lenguaje } & $\mathrm{C}$ de Dunnett & Grad Alto & Grad Bajo & $-57,70$ & No SIG. \\
\hline & & Grad Alto & Grad Medio & $-49,35$ & No SIG. \\
\hline & & Grad Medio & Grad Bajo & $-8,35$ & No SIG. \\
\hline & & NS Alto & NS Bajo & $-71,39$ & SIG. \\
\hline & & NS Alto & NS Medio & $-21,74$ & No SIG. \\
\hline & & NS Medio & NS Bajo & $-49,65$ & SIG. \\
\hline & & Ret Alto & Ret Bajo & $-34,35$ & No SIG. \\
\hline & & Ret Alto & Ret Medio & $-6,11$ & No SIG. \\
\hline & & Ret Medio & Ret Bajo & $-28,24$ & No SIG. \\
\hline \multirow[t]{9}{*}{ Matemáticas } & Bonferroni & Grad Alto & Grad Bajo & $-85,33$ & ,096 \\
\hline & & Grad Alto & Grad Medio & $-54,56$ & 1,000 \\
\hline & & Grad Medio & Grad Bajo & $-30,77$ & 1,000 \\
\hline & & NS Alto & NS Bajo & $-78,96$ &, 000 \\
\hline & & NS Alto & NS Medio & $-28,53$ & ,008 \\
\hline & & NS Medio & NS Bajo & $-50,43$ &, 000 \\
\hline & & Ret Alto & Ret Bajo & $-32,97$ & 1,000 \\
\hline & & Ret Alto & Ret Medio & $-7,91$ & 1,000 \\
\hline & & Ret Medio & Ret Bajo & $-25,07$ & 1,000 \\
\hline Historia & Bonferroni & Grad Alto & Grad Bajo & $-82,16$ & ,891 \\
\hline
\end{tabular}


Estudios Pedagógicos XLIII, N 1: 171-191, 2017

TALENTO ACADÉMICO, VULNERABILIDAD ESCOLAR Y RESULTADOS EN LA PRUEBA DE SELECCIÓN UNIVERSITARIA

\begin{tabular}{|c|c|c|c|c|c|}
\hline & & Grad Alto & Grad Medio & $-43,52$ & 1,000 \\
\hline & & Grad Medio & Grad Bajo & $-38,64$ & 1,000 \\
\hline & & NS Alto & NS Bajo & $-67,40$ & ,000 \\
\hline & & NS Alto & NS Medio & $-26,16$ & ,294 \\
\hline & & NS Medio & NS Bajo & $-41,24$ & ,003 \\
\hline & & Ret Alto & Ret Bajo & $-20,17$ & 1,000 \\
\hline & & Ret Alto & Ret Medio & 10,83 & 1,000 \\
\hline & & Ret Medio & Ret Bajo & $-31,00$ & 1,000 \\
\hline \multirow[t]{9}{*}{ Ciencias } & Bonferroni & Grad Alto & Grad Bajo & $-132,21$ & ,006 \\
\hline & & Grad Alto & Grad Medio & $-80,82$ & ,916 \\
\hline & & Grad Medio & Grad Bajo & $-51,39$ & 1,000 \\
\hline & & NS Alto & NS Bajo & $-65,11$ & ,000 \\
\hline & & NS Alto & NS Medio & $-21,61$ & 1,000 \\
\hline & & NS Medio & NS Bajo & $-43,51$ & ,000 \\
\hline & & Ret Alto & Ret Bajo & $-80,47$ &, 010 \\
\hline & & Ret Alto & Ret Medio & $-17,42$ & 1,000 \\
\hline & & Ret Medio & Ret Bajo & $-63,05$ & ,088 \\
\hline \multirow[t]{9}{*}{ NEM } & Bonferroni & Grad Alto & Grad Bajo & $-19,72$ & 1,000 \\
\hline & & Grad Alto & Grad Medio & $-48,65$ & 1,000 \\
\hline & & Grad Medio & Grad Bajo & 28,94 & 1,000 \\
\hline & & NS Alto & NS Bajo & $-16,41$ & 1,000 \\
\hline & & NS Alto & NS Medio & $-3,85$ & 1,000 \\
\hline & & NS Medio & NS Bajo & $-12,56$ & 1,000 \\
\hline & & Ret Alto & Ret Bajo & $-13,39$ & 1,000 \\
\hline & & Ret Alto & Ret Medio & $-2,91$ & 1,000 \\
\hline & & Ret Medio & Ret Bajo & $-10,48$ & 1,000 \\
\hline
\end{tabular}

En los alumnos Graduados solamente se encontraron diferencias significativas en la PSU Ciencias, entre los grupos de baja vulnerabilidad y de alta vulnerabilidad. En las otras PSU no se observaron diferencias significativas según vulnerabilidad en los estudiantes que finalizaron el programa de talentos. Situación análoga ocurrió en el grupo de estudiantes Retirados, en el que no se encontraron diferencias significativas según vulnerabilidad, con excepción de la PSU Ciencias entre los alumnos de nivel bajo y nivel alto de vulnerabilidad. En los estudiantes No Seleccionados se observaron diferencias significativas en todas las PSU, por un lado, entre los alumnos de nivel bajo y nivel medio de vulnerabilidad, y por otro, entre los grupos de nivel bajo y nivel alto. Además, en el caso de los No Seleccionados, 
las diferencias resultaron significativas también entre los estudiantes de nivel medio y nivel alto de vulnerabilidad en la PSU Matemática.

Con excepción de la PSU Ciencias, la tendencia de estos resultados parece indicar que el nivel de vulnerabilidad de los estudiantes no influiría en los resultados PSU cuando el estudiante es identificado como con potencial de talento académico y participa (parcial o completamente) en un programa especialmente diseñado para el desarrollo de dicho talento. Esto mostraría que la participación en el programa de talentos permite poner en igualdad de condiciones a los estudiantes con altas habilidades cognitivas independientemente de su vulnerabilidad o, en todo caso, contribuir a desarrollar herramientas para disminuir las brechas dadas por los contextos de origen. Por el contrario, esto no se observa en los estudiantes No Seleccionados, donde las diferencias asociadas a la vulnerabilidad de sus escuelas se relacionan con los puntajes PSU, lo que refuerza la evidencia previa respecto al sesgo socioeconómico de la PSU.

Al comparar las notas de educación media según vulnerabilidad, no se encontraron diferencias dentro de ninguno de los grupos de participación en PentaUC. Esto mostraría que en todos los grupos socioeconómicos se encuentran estudiantes que aprovechan de buena forma las oportunidades académicas.

\subsection{COMPARACIONES SEGÚN NIVEL DE PARTICIPACIÓN EN PENTAUC}

Para cada una de las pruebas de selección universitaria se compararon los grupos según participación en el Programa PentaUC dentro de cada nivel de vulnerabilidad escolar (ver Tabla 6). Este análisis permite aproximarse a dos fenómenos: i) la influencia de la participación completa o parcial en un programa de talentos en los resultados PSU en cada nivel de vulnerabilidad (comparando a graduados con retirados) y ii) la influencia del potencial de talento académico en los resultados PSU dentro de cada nivel de vulnerabilidad (comparando a no seleccionados con los otros dos grupos).

Tabla 6. Pruebas Post Hoc según participación dentro de cada nivel de vulnerabilidad

\begin{tabular}{|l|l|c|c|c|c|}
\hline PSU & Prueba Post Hoc & Grupo (I) & Grupo (J) & $\begin{array}{c}\text { Diferencia de } \\
\text { medias (I-J) }\end{array}$ & $\begin{array}{c}\text { Nivel de } \\
\text { significancia }\end{array}$ \\
\hline Lenguaje & C de Dunnett & Alto Grad & Alto NS & 90,784 & SIG. \\
\hline & & Alto Grad & Alto Ret & 26,767 & No SIG. \\
\hline & & Alto Ret & Alto NS & 64,017 & SIG. \\
\hline & & Bajo Grad & Bajo NS & 77,092 & SIG. \\
\hline & & Bajo Grad & Bajo Ret & 50,114 & SIG. \\
\hline & & Bajo Ret & Bajo NS & 26,979 & No SIG. \\
\hline & & Medio Grad & Medio NS & 118,389 & SIG. \\
\hline & & Medio Grad & Medio Ret & 70,000 & SIG. \\
\hline
\end{tabular}


Estudios Pedagógicos XLIII, N ${ }^{\circ}$ : 171-191, 2017

TALENTO ACADÉMICO, VULNERABILIDAD ESCOLAR Y RESULTADOS EN LA PRUEBA DE SELECCIÓN UNIVERSITARIA

\begin{tabular}{|c|c|c|c|c|c|}
\hline \multirow[t]{9}{*}{ Matemáticas } & Bonferroni & Alto Grad & Alto NS & 98,130 & ,003 \\
\hline & & Alto Grad & Alto Ret & 16,876 & 1,000 \\
\hline & & Alto Ret & Alto NS & 81,254 & ,000 \\
\hline & & Bajo Grad & Bajo NS & 104,501 & ,000 \\
\hline & & Bajo Grad & Bajo Ret & 69,229 & ,003 \\
\hline & & Bajo Ret & Bajo NS & 35,272 &, 032 \\
\hline & & Medio Grad & Medio NS & 124,162 & ,000 \\
\hline & & Medio Grad & Medio Ret & 63,530 & ,158 \\
\hline & & Medio Ret & Medio NS & 60,632 & ,001 \\
\hline \multirow[t]{9}{*}{ Historia } & Bonferroni & Alto Grad & Alto NS & 66,434 & 1,000 \\
\hline & & Alto Grad & Alto Ret & 12,800 & 1,000 \\
\hline & & Alto Ret & Alto NS & 53,634 & ,888 \\
\hline & & Bajo Grad & Bajo NS & 81,192 & ,014 \\
\hline & & Bajo Grad & Bajo Ret & 74,784 & ,099 \\
\hline & & Bajo Ret & Bajo NS & 6,408 & 1,000 \\
\hline & & Medio Grad & Medio NS & 83,798 & ,013 \\
\hline & & Medio Grad & Medio Ret & 67,149 & ,623 \\
\hline & & Medio Ret & Medio NS & 16,649 & 1,000 \\
\hline \multirow[t]{9}{*}{ Ciencias } & Bonferroni & Alto Grad & Alto NS & 39,776 & 1,000 \\
\hline & & Alto Grad & Alto Ret & $-5,494$ & 1,000 \\
\hline & & Alto Ret & Alto NS & 45,270 & ,966 \\
\hline & & Bajo Grad & Bajo NS & 106,870 & ,000 \\
\hline & & Bajo Grad & Bajo Ret & 46,241 & 1,000 \\
\hline & & Bajo Ret & Bajo NS & 60,629 &, 000 \\
\hline & & Medio Grad & Medio NS & 98,990 &, 000 \\
\hline & & Medio Grad & Medio Ret & 57,907 & ,958 \\
\hline & & Medio Ret & Medio NS & 41,083 & ,985 \\
\hline \multirow[t]{9}{*}{ NEM } & Bonferroni & Alto Grad & Alto NS & 59,767 & 1,000 \\
\hline & & Alto Grad & Alto Ret & 31,255 & 1,000 \\
\hline & & Alto Ret & Alto NS & 28,512 & 1,000 \\
\hline & & Bajo Grad & Bajo NS & 63,068 & ,013 \\
\hline & & Bajo Grad & Bajo Ret & 37,578 & 1,000 \\
\hline & & Bajo Ret & Bajo NS & 25,490 & 1,000 \\
\hline & & Medio Grad & Medio NS & 104,569 & ,000 \\
\hline & & Medio Grad & Medio Ret & 76,994 & 057 \\
\hline & & Medio Ret & Medio NS & 27,575 & 1,000 \\
\hline
\end{tabular}


Al analizar los resultados de Graduados y Retirados se observó que en el nivel bajo de vulnerabilidad las diferencias fueron significativas en la PSU Matemática y en la PSU Lenguaje. En esta última, también se observaron diferencias significativas en el nivel medio de vulnerabilidad. En la PSU Historia y la PSU Ciencias no se encontraron diferencias entre Graduados y Retirados en ninguno de los niveles de vulnerabilidad.

Que las diferencias entre Graduados y Retirados se presenten únicamente en las PSU Lenguaje y PSU Matemáticas pareciera indicar que la participación total en un programa de desarrollo del talento académico tiene algún impacto en el desarrollo de habilidades, competencias y/o contenidos necesarios para rendir de buena forma dichas PSU. Dado que el programa PentaUC es extracurricular, es menos probable que dicho impacto se encuentre en la adquisición de contenidos y, por lo tanto, tenga más que ver con adquisición de competencias para abordar dichos contenidos en los procesos de preparación de la PSU y con habilidades lingüísticas y lógico matemáticas que pudieran influir en la resolución de las pruebas.

La falta de diferencias en PSU Lenguaje y PSU Matemáticas entre los alumnos de alta vulnerabilidad que participaron parcialmente en el programa y aquellos de alta vulnerabilidad que participaron totalmente parece indicar que completar el programa para estos alumnos no hace diferencia en su resultado PSU. Como hipótesis, se puede decir que los estudiantes de mayor vulnerabilidad parecen tener más dificultades para hacer un aprovechamiento máximo del Programa aun cuando lo finalizan; también puede implicar que la brecha que traerían (en relación a los estudiantes de menor vulnerabilidad) requeriría más tiempo en el Programa, u otras medidas, para que la compensación sea completa y represente un impacto en el puntaje PSU. Estas hipótesis y posibles alternativas deben ser investigadas en el futuro.

La ausencia de diferencias entre Graduados y Retirados en todos los niveles de vulnerabilidad en las PSU Historia y PSU Ciencias muestra que el impacto de la participación completa o parcial en el programa de talentos no es significativa en pruebas de áreas centradas en medición de conocimientos. Cabe recordar que el Programa PentaUC es extracurricular y, por lo tanto, no pretende enseñar ni reforzar contenidos escolares.

Para analizar la influencia del potencial de talento académico en los resultados PSU, dentro de cada nivel de vulnerabilidad, se compararon los resultados de los estudiantes No Seleccionados con los otros dos grupos. En todas las PSU (excepto en Historia y Ciencias, en los alumnos de vulnerabilidad Alta) se observaron diferencias significativas entre el grupo de Graduados y el grupo de No Seleccionados. El grupo de estudiantes Retirados mostró diferencias significativas con los No Seleccionados en la PSU Lenguaje (en nivel medio y alto de vulnerabilidad), en la PSU Matemáticas (en todos los niveles de vulnerabilidad) y en la PSU Ciencias (nivel bajo de vulnerabilidad).

En conjunto, las comparaciones entre estudiantes identificados con potencial talento académico y estudiantes sin esa identificación mostrarían que el potencial de talento académico establece una diferencia significativa en los resultados PSU (principalmente en Matemática y Lenguaje). Sin embargo, es aún necesario determinar si estas diferencias se establecen debido a las altas habilidades cognitivas por sí mismas, o bien por la identificación del talento y la participación (parcial o completa) en un programa especial. Dadas las características de la presente muestra, la segunda alternativa cobra mayor relevancia, ya que la participación en este tipo de programas no solamente implica la identificación del potencial de talento, sino que apunta a reforzar sus características 
personales y académicas, movilizar el entorno familiar, el entorno escolar y generar la construcción de altas expectativas (Narea, Lissi \& Arancibia, 2006), todas las cuales actúan como catalizadores para el desarrollo del talento (Gagné, 2004).

La situación de los alumnos Retirados requiere una mirada más profunda, debido a que, según los resultados expuestos, los puntajes de estos estudiantes en unos casos son comparables con los de los alumnos graduados y, en otros, con los de los alumnos No Seleccionados. Este grupo debiera estudiarse en función de otras variables, como por ejemplo la cantidad de años de participación en el Programa y los motivos de los retiros. Este esfuerzo no se llevó a cabo en el presente estudio debido a que una clasificación de ese tipo hubiese reducido excesivamente el tamaño de los grupos y, por lo tanto, afectado los análisis.

Al comparar las notas de enseñanza media (NEM) solamente se observaron diferencias significativas en los niveles bajo y medio de vulnerabilidad, entre los grupos de Graduados y No Seleccionados. Esto pareciera señalar un impacto en los rendimientos escolares a favor de aquellos estudiantes con potencial talento académico que terminan el programa especial en estos niveles de vulnerabilidad.

\subsection{ANÁLISIS DE REGRESIÓN}

Para cada grupo de participación en el Programa (No Seleccionados, Retirados y Graduados) se evaluó el poder predictivo de las variables Notas de Enseñanza Media (NEM), Vulnerabilidad Escolar (IVE) y Habilidades Cognitivas (medidas con el test de Raven) respecto de los puntajes PSU en cada uno de los niveles de participación en PentaUC.

Como se dijo antes, para este análisis se utilizó el IVE en su forma estandarizada (como variable continua) y no como clasificación grupal, es por ello que se calcularon las medias para cada grupo de participación en el Programa, pero esta vez sin dividirlos según vulnerabilidad escolar (ver tablas 7 y 8). La prueba ANOVA y los análisis post hoc mostraron que en todas las PSU, y en el NEM, las diferencias son significativas entre los tres grupos (PSU Lenguaje: F $(2,1001)=61.86$, p $\leq .001$; PSU Matemáticas: $F(2,1001)=81.63$, $\mathrm{p} \leq .001$; PSU Historia: $\mathrm{F}(8,599)=20.30, \mathrm{p} \leq .001$; PSU Ciencias: $\mathrm{F}(2,562)=39.13, \mathrm{p} \leq .001$; NEM: $F(2,1063)=26.57, p \leq .001)$, mostrando que el resultado de los Graduados es siempre el mayor y el de los No Seleccionados siempre el menor.

Tabla 7. Promedios PSU Lenguaje y Matemática según Participación en PENTA UC

\begin{tabular}{|l|c|c|c|c|}
\hline \multirow{2}{*}{ Participación en PENTA UC } & \multicolumn{2}{|c|}{ PSU Lenguaje } & \multicolumn{2}{c|}{ PSU Matemáticas } \\
\cline { 2 - 5 } & Promedio & $\mathrm{n}$ & Promedio & $\mathrm{n}$ \\
\hline No Seleccionados & 529.58 & 768 & 516.11 & 769 \\
\hline Retirados & 583.1 & 165 & 581.68 & 164 \\
\hline Graduados & 633.46 & 71 & 637.54 & 71 \\
\hline
\end{tabular}


Tabla 8. Promedios PSU Historia, Ciencias y Puntaje NEM según Participación en PENTA UC

\begin{tabular}{|l|c|c|c|c|c|c|}
\hline \multirow{2}{*}{ Participación en PENTA UC } & \multicolumn{2}{|c|}{ PSU Historia } & \multicolumn{2}{c|}{ PSU Ciencias } & \multicolumn{2}{c|}{ NEM } \\
\cline { 2 - 7 } & Promedio & $\mathrm{n}$ & Promedio & $\mathrm{n}$ & Promedio & $\mathrm{n}$ \\
\hline No Seleccionados & 536.24 & 469 & 525.5 & 414 & 563.42 & 826 \\
\hline Retirados & 568.86 & 91 & 585.43 & 100 & 593.44 & 169 \\
\hline Graduados & 623.5 & 42 & 624.45 & 51 & 643.24 & 71 \\
\hline
\end{tabular}

La Tabla 9 muestra las varianzas explicadas y los coeficientes beta de cada modelo probado en las regresiones simples.

Tabla 9. Coeficientes y varianza explicada para cada PSU

\begin{tabular}{|c|l|c|c|c|}
\hline Var. Dependiente & \multicolumn{1}{|c|}{ Coeficientes } & No Seleccionados & Retirados & Graduados \\
\hline \multirow{5}{*}{ PSU Lenguaje } & Varianza Explicada & $30,3 \%$ & $29,2 \%$ & $6,9 \%$ \\
\cline { 2 - 5 } & NEM (Beta) & 0.376 & 0.46 & \\
\cline { 2 - 5 } & IVE (Beta) & -0.265 & -0.193 & -0.287 \\
\cline { 2 - 5 } & RAVEN (Beta) & 0.201 & 0.172 & \\
\hline \multirow{5}{*}{ PSU Matemáticas } & Varianza Explicada & $31,6 \%$ & $29,1 \%$ & $23,5 \%$ \\
\cline { 2 - 5 } & NEM (Beta) & 0.381 & 0.471 & 0.29 \\
\cline { 2 - 5 } & IVE (Beta) & -0.256 & -0.178 & -0.322 \\
\cline { 2 - 5 } & RAVEN (Beta) & 0.218 & 0.163 & \\
\hline & Varianza Explicada & $21 \%$ & $14,6 \%$ & $0 \%$ \\
\cline { 2 - 5 } & NEM (Beta) & 0.276 & 0.395 & \\
\cline { 2 - 5 } & IVE (Beta) & -0.216 & & $31,6 \%$ \\
\cline { 2 - 5 } & RAVEN (Beta) & 0.202 & & -0.442 \\
\hline \multirow{5}{*}{ PSU Ciencias } & Varianza Explicada & $22,3 \%$ & $32,4 \%$ & \\
\cline { 2 - 5 } & NEM (Beta) & 0.369 & 0.395 & \\
\cline { 2 - 5 } & IVE (Beta) & -0.256 & -0.35 & \\
\cline { 2 - 5 } & RAVEN (Beta) & 0.107 & & \\
\hline
\end{tabular}

En relación con los factores incluidos en los modelos se observa que las variables que explican los distintos resultados PSU varían según el grupo de Participación que se observe.

En el Grupo de No Seleccionados siempre aparecen los tres factores, observándose que el NEM tiene el mayor peso en todas las Pruebas y el RAVEN, el menor. Asimismo, los modelos poseen el mayor porcentaje de varianza explicada en PSU Lenguaje, PSU Matemáticas y PSU Historia. 
En los Retirados, las PSU Lenguaje y PSU Matemáticas mantienen el patrón del grupo anterior; sin embargo, en la PSU Historia las variables IVE y RAVEN no resultaron significativas; mientras que en la PSU Ciencias ocurrió lo propio con la variable RAVEN.

Finalmente, en el grupo de Graduados todos los modelos excluyen la variable RAVEN, y en el caso de la PSU Lenguaje y PSU Historia, también el NEM. En este grupo ninguna de las variables fue significativa para el modelo explicativo de la PSU Historia.

Estos resultados dan cuenta de un panorama complejo respecto de la relación de los resultados PSU, las habilidades cognitivas (medidas con el test de Raven), el rendimiento académico, el nivel de vulnerabilidad y la participación en un programa de talentos académicos.

El primer punto a observar es el comportamiento de la variable RAVEN. Esta variable representa a la inteligencia general y las habilidades analíticas. Su ausencia dentro del grupo de los Graduados implicaría que la inteligencia general no explica las diferencias en sus puntajes PSU, a diferencia de lo que ocurre en los No Seleccionados. Esto se podría entender por el hecho de que la PSU, en términos generales, no busca medir habilidades cognitivas generales, sino la aplicación de contenidos curriculares, por lo que la obtención de altos puntajes PSU (como los observados en este grupo) no requeriría de la participación directa de estas habilidades. En relación con los alumnos Retirados surgen importantes preguntas, pues en ellos la variable RAVEN solamente aparece en las PSU Lenguaje y PSU Matemática. Pese a que no existen diferencias significativas en sus puntajes RAVEN comparados con el de los Graduados (ambos grupos fueron seleccionados en PentaUC), los modelos difieren en cuanto a la inclusión de la inteligencia general para estas dos PSU. Se puede pensar que el hecho de terminar el programa contribuye de una manera tal que permitiría el desarrollo de competencias que ayudan a alcanzar altos puntajes PSU, las cuales tendrían más valor predictivo que las habilidades cognitivas evaluadas con el test de Raven.

La variable NEM tiene participación en la mayoría de los modelos, dando cuenta de que el aprendizaje de contenidos en el colegio y la capacidad del estudiante para responder a las exigencias escolares son dimensiones que explican las variaciones en los puntajes PSU. Por otro lado, la variable NEM desaparece de los modelos explicativos de las PSU Lenguaje y PSU Historia, grupo de Graduados, lo que significa que las diferencias de NEM entre los graduados no explican sus diferencias en los puntajes PSU de estas pruebas. Esto podría implicar que para obtener altos puntajes en esas dos pruebas el estudiante debe desarrollar estrategias que no serían exactamente las mismas para rendir bien en el colegio. La posibilidad de desarrollar estas estrategias las facilitaría la participación completa en un programa de talentos, puesto que en los Retirados el factor NEM sigue apareciendo.

Con relación al IVE, los resultados de las regresiones entregan información que ratifica la relación inversa entre vulnerabilidad y resultados PSU en las Pruebas de Matemáticas, Lenguaje y Ciencias.

Al mirar cada una de las PSU por separado también se observan relaciones complejas entre las variables de estudio. El resultado PSU Lenguaje obtenido por estudiantes con potencial de talento académico que han finalizado el Programa no se explicaría por las habilidades cognitivas ni por el desempeño escolar. Esto podría ser entendido por el hecho de que los estudiantes que finalizan el Programa han desarrollado estrategias más eficientes para abordar la PSU Lenguaje y sus contenidos, estrategias que no son las mismas que se requieren para tener éxito en el colegio (en donde ellos muestran también los mejores resultados) ni implican la utilización directa de las habilidades cognitivas, pues estas 
últimas, como se sabe, por el hecho de haber sido identificados como estudiantes con potencial talento académico, poseen en un alto nivel en relación a sus pares.

En el resultado PSU Matemáticas se pueden obtener conclusiones similares en lo referente a las habilidades cognitivas. Sin embargo, respecto del NEM, su presencia en la ecuación de los estudiantes graduados indicaría que las estrategias y características personales utilizadas para rendir bien en el colegio son aplicables en la PSU Matemáticas y resultan relevantes tanto en estudiantes No Seleccionados, como en estudiantes identificados como con potencial talento académico.

Lo observado en la PSU Historia puede explicarse desde el punto de vista del contenido de dicha prueba. Esta prueba es la que más contenidos evalúa, es por ello que en los resultados de los estudiantes identificados como con potencial de talento es probable que un factor no evaluado aquí, del tipo estrategias de estudio o interés, esté teniendo mayor importancia. Cabe notar que, como en el caso de PSU Lenguaje, para obtener altos resultados en la PSU Historia, las estrategias utilizadas en el colegio no parecen ser suficientes.

La PSU Ciencias muestra una mayor incidencia del factor Vulnerabilidad, por lo que podría suponerse que, incluso existiendo participación en un Programa de Talentos, la calidad del entorno educativo escolar va a tener un mayor peso. Puede suponerse que para este tipo de contenidos, a diferencia de los contenidos en historia, la calidad del entorno académico escolar es factor importante para obtener altos resultados, pese a la presencia del apoyo que entregue el Programa de Talentos. Por otro lado, la interpretación de los resultados observados en esta PSU se ve dificultada porque esta prueba no es equivalente en todos los alumnos, pues existe un módulo optativo que los estudiantes escogen (que representa el 32,5\% de la prueba) y cuyo contenido es distinto. En este sentido, el Informe Pearson (Pearson, 2013) señala, por ejemplo, que es un error metodológico entregar un puntaje único para una prueba que incluye preguntas de biología, física y química y no en la misma proporción (por la existencia del módulo electivo).

En términos globales, se observa que los Graduados presentan el menor porcentaje de varianza explicada por el modelo propuesto en todas las pruebas, excepto en la PSU de Ciencias. Considerando que estos alumnos obtienen los más altos puntajes PSU de la muestra, puede hipotetizarse que los factores del modelo propuesto (NEM, RAVEN e IVE) explican un porcentaje menor de las diferencias de puntaje entre los sujetos que se ubican en el extremo superior de la escala PSU (sobre 1 desviación estándar, según los datos observados). En otras palabras, las variables que explican las diferencias entre dos puntajes altos de la escala son diferentes a las que explican las diferencias entre dos puntajes medios o bajos en la PSU Lenguaje, PSU Matemáticas y PSU Historia. Se puede pensar que aquellas variables estarían relacionadas con catalizadores intrapersonales que el Programa de Talentos ayudaría a desarrollar en los estudiantes, así como con la motivación y capacidad para organizar tiempos y enfrentar múltiples exigencias. Esto es materia de futuras investigaciones.

\section{CONCLUSIONES Y DISCUSIÓN}

En el contexto chileno, la posibilidad de contribuir a disminuir las diferencias de oportunidades académicas asociadas a sesgos socioeconómicos resulta ser un área de primera prioridad. El presente estudio ha tenido la intención de poder ahondar en una temática no tra- 
tada previamente y que puede tener implicancias prácticas a este respecto: la relación entre talento académico, vulnerabilidad escolar y rendimiento en PSU. Los resultados mostraron relaciones complejas entre las variables y dejaron abiertas nuevas líneas de investigación.

Se mostró que los estudiantes con potencial de talento obtienen mejores resultados PSU que aquellos sin dicho potencial. A su vez, quienes completan un Programa de Talentos (en este caso 7 años de participación) obtienen mejores resultados PSU que quienes lo abandonan. Se observó que cuando un estudiante es identificado con potencial de talento y puede participar de una instancia académica adecuada a sus necesidades, las diferencias en los puntajes PSU que se esperarían en consideración de la vulnerabilidad de sus escuelas de origen tienden a disminuir o desaparecer.

Al mismo tiempo, cuando se estudian estas relaciones a la luz del nivel de vulnerabilidad de los estudiantes, se observa que dicho nivel genera que el efecto del potencial talento académico (altas habilidades cognitivas) y el efecto de la participación en un programa especial sobre los puntajes PSU varían.

Se observó que la participación completa o parcial en un programa de talentos genera diferencias solamente en niveles medios y bajos de vulnerabilidad en los resultados de la PSU Matemáticas y la PSU Lenguaje, no detectándose dichas diferencias en el nivel alto de vulnerabilidad. Pareciera ser entonces que, cuando el estudiante proviene de un contexto educativo de alta vulnerabilidad, la posibilidad de que él aproveche al máximo la instancia ofrecida y/o que el programa le entregue suficientes herramientas para compensar la influencia de la vulnerabilidad contextual en los resultados PSU no logran un ajuste completo, lo que obliga a buscar, en futuras investigaciones, por dónde pasan esos ajustes y cómo poder implementarlos.

En las PSU Historia y PSU Ciencias en ningún nivel de vulnerabilidad se observaron diferencias entre finalizar o abandonar el programa de talentos. Estos resultados pueden vincularse con los tipos de contenidos y habilidades evaluados en cada PSU, los que podrían modular el impacto de las variables revisadas sobre los puntajes obtenidos. Además, cabe señalar que el Programa de Talentos estudiado tiene el foco en desarrollar habilidades cognitivas específicas, sin implicar un refuerzo de los contenidos curriculares obligatorios.

Se observó que cuando los estudiantes son identificados como con potencial talento académico logran establecer claras diferencias en sus resultados PSU Lenguaje y Matemáticas, en cualquier nivel de vulnerabilidad, con relación a sus pares no identificados, pero no así en las PSU Historia y PSU Ciencias en los niveles altos de vulnerabilidad.

Por último, se vio que para alcanzar altos puntajes en la PSU no basta con las habilidades cognitivas y, en algunos casos, tampoco basta con replicar el modelo de estudio y abordaje de contenidos que tiene resultados en el colegio. Queda pendiente la exploración detallada de estas características, las cuales pueden estar asociadas con procesos autorregulatorios y meta cognitivos que contribuyen a abordar eficientemente los procesos de aprendizaje.

A modo de conclusión, se puede decir que los estudiantes con potencial talento académico obtienen mejores resultados en la PSU debido a que las habilidades cognitivas, y su participación en un Programa de Talentos, favorecen el desarrollo de catalizadores intrapersonales que contribuyen al aprendizaje significativo de los conocimientos para rendir la PSU, y que en algunos casos pueden ser diferentes a los que se requieren para rendir bien en sus colegios. Este efecto, según los datos analizados, no llegaría de la misma forma a todos los niveles de vulnerabilidad, por lo que se abre un importante desafío futuro a nivel de investigación y a nivel práctico. 
Para finalizar, es necesario mencionar algunas de las limitaciones del presente estudio. En primer lugar, debido a la muestra utilizada, que implicó únicamente 4 años de procesos completos de estudiantes en PentaUC, los números de sujetos por grupo (principalmente en los graduados de vulnerabilidad alta) resultaron ser muy pequeños, por lo que algunos de los resultados pudieron estar influidos por dicho factor. Es importante replicar los análisis realizados con muestras mayores.

En segundo lugar, se debe considerar la heterogeneidad del grupo de los estudiantes Retirados: este es un grupo que incluye sujetos con distintos años de participación en el Programa y distintos motivos de retiros. Ambas dimensiones pueden tener un efecto en los resultados obtenidos, por lo que es altamente recomendable, cuando el número de sujetos de la población lo permita, realizar estudios que incluyan subclasificaciones dentro de los alumnos que han abandonado el Programa.

En tercer lugar, se observó que los modelos probados mediante las regresiones explicaron, en general, bajos porcentaje de la varianza observada. Es una tarea futura incorporar nuevas variables a los modelos, para así robustecerlos y obtener hipótesis más comprehensivas del fenómeno.

Finalmente, habida cuenta de la extensa bibliografía existente sobre las diferencias de resultados académicos según sexo, es importante diseñar futuras investigaciones que consideren dicha variable y su interacción con aquellas revisadas en el presente estudio.

\section{REFERENCIAS BIBLIOGRÁFICAS}

ACT. (2007). The ACT: Technical manual. Iowa City: ACT.

Arancibia, V. (1992). Efectividad escolar: Un análisis comparado. Estudios Públicos, 47, 101-125.

Arancibia, V. (2009). La educación de alumnos con talentos: una deuda y una oportunidad para Chile. Temas de la agenda pública: Vicerrectoría de Comunicaciones y Asuntos Públicos, Pontificia Universidad Católica de Chile, 26, 3-15.

Arancibia, V., \& González, S. (2007). Modelo PENTA UC. Guía para implementar un programa para niños, niñas y jóvenes con talentos académicos en la universidad. Santiago: Pontificia Universidad Católica de Chile.

Beaujean, A. A., Firmin, M. W., Knoop, A. J., Michonski, J. D., Berry, T. B., \& Lowrie, R. E. (2006). Validation of the Frey \& Detterman (2004) IQ prediction equations using the Reynolds Intellectual Assessment Scales. Personality \& Individual Differences, 41(2), 353-357. doi:10.1016/j.paid.2006.01.014

Benavides, M., Ríos, C. G., \& Marshal, M. V. (2004). La educación de niños con talento en Chile. En M. Benavides, A. Maz, E. Castro \& R. Blanco (Eds.), La educación de Niños con Talento en Iberoamérica (pp. 105-114). Santiago de Chile: Trineo.

Beyer, H. (2009). Igualdad de oportunidades y selección a las universidades. Puntos de Referencia, $303,1-10$.

Bravo, D., del Pini, G., Donoso, G., Manzi, J., Martínez, M., \& Pizarro, R. (2008). Resultados de la aplicación de la prueba de selección universitaria, admisión 2006-2008. Santiago: Documentos Técnicos Honorable Consejo de Rectores de las Universidades Chilenas.

Brody, L., \& Benbow, C.P. (1990). Effects of high school coursework \& time on SAT scores. Journal of Educational Psychology, 82(4), 866-875. doi:10.1037/0022-0663.82.4.866

Centro de Políticas Públicas UC. (2009). Políticas de educación superior: reflexiones y desafíos para una reforma al sistema. Santiago: Pontificia Universidad Católica de Chile.

Coleman, J. S. (1988). Social capital in the creation of human capital. American Journal of Sociology, 
Estudios Pedagógicos XLIII, N 1: 171-191, 2017

TALENTO ACADÉMICO, VULNERABILIDAD ESCOLAR Y RESULTADOS EN LA PRUEBA DE SELECCIÓN

UNIVERSITARIA

94, 95-120.

Cornejo, A., Céspedes, P., Escobar, D., Núñez, R., Reyes, G., \& Rojas, K. (2005). SINAE, Sistema nacional de asignación con equidad para becas JUNAEB. Una nueva visión en la construcción de igualdad de oportunidades en la infancia. Santiago: Junta Nacional de Auxilio Escolar y Becas.

DEMRE. (2005). Teoría clásica de la medición TC y teoría de la respuesta al ítem TRI. Santiago: Autor.

Dussaillant, F. (2003). Técnicas de medición en pruebas de admisión a las universidades. Estudios Públicos, 90, 91-144.

Freeman, J. (2005). Permission to be gifted: how conceptions of giftedness can change lives. In R. Sternberg \& J. Davidson (Eds.), Conceptions of Giftedness (pp. 80-97). Cambridge: Cambridge University Press.

Frey, M., \& Detterman, D. (2004). Scholastic assessment or g? The relationship between the Scholastic Assessment Test \& general cognitive ability. Psychological Science, 15(6), 373-378. doi:10.1111/j.0956-7976.2004.00687.x

Gagné, F. (2004). Transforming gifts into talents: the DMGT as a developmental theory. High Ability Studies, 15(2), 119-147. doi:10.1080/1359813042000314682

González, M., \& Glavic, M. (2011). Impacto de la educación de talentos académicos en contextos sociales y económicos vulnerables. XXVIII Congreso internacional de ALAS. Recife.

JUNAEB.(2012). Base de dato extraída de: http://www.junaeb.cl/prontus_junaeb/site/artic/20100118/ pags/20100118133326.html

Koenig, K. A., Frey, M. C., \& Detterman, D. K. (2008). ACT and general cognitive ability. Intelligence, 36(2), 153-160. doi:10.1016/j.intell.2007.03.005

Koljatic, M., \& Silva, M. (2010). Algunas reflexiones a siete años de la implementación de la PSU. Estudios públicos, 120, 125-146.

Meckes, L., \& Bascopé, M. (2009). Distribución inequitativa de los nuevos profesores mejor preparados: Características de origen y destino laboral de los egresados de pedagogía básica. Santiago: Centro de estudios de políticas y prácticas en educación.

Narea, M., Lissi, M., \& Arancibia, V. (2006). Impacto en la sala de clases de un programa extraescolar de enriquecimiento para alumnos con talentos académicos. Psykhe, 15(2), 81-92. doi:10.4067/ S0718-22282006000200008

OECD., World Bank. (2009). La educación superior en Chile. Revisión de Políticas Nacionales de Educación. Santiago: Ministerio de Educación.

Ortúzar, M., Flores, S., Milesi, C., \& Cox, C. (2009). Aspectos de la formación inicial docente y su influencia en el rendimiento académico de los alumnos. En I. Irarrázaval, E. Puga \& M. Letelier (Eds.), Camino al Bicentenario. Propuestas para Chile (pp. 155-186). Santiago: Pontificia Universidad Católica de Chile.

Paredes, R., \& Paredes, V. (2009). Chile: rendimiento académico y gestión de la educación en un contexto de rigidez laboral. Revista de la CEPAL, 99, 119-131.

Pearson (2013). Informe Final Evaluación de la PSU Chile. London: Pearson.

Plank, S. B., \& Jordan, W. J. (2001). Effects of information, guidance \& actions on postsecondary destinations: a study of talent loss. American Educational Research Journal, 38(4), 947-979. doi: $10.3102 / 00028312038004947$

Raven, J.C., Court, J.H., \& Raven, J. (1996). Test de matrices progresivas. Escalas coloreada, general y avanzada. Buenos Aires: Paidós.

Renzulli, J. (2012). Reexamining the role of gifted education and talent development for the 21st century: a four-part theoretical approach. Gifted Child Quarterly, 56(3), 150-159. doi:10.1177/0016986212444901

Rohde, T. E., \& Thompson, L. A. (2007). Predicting academic achievement with cognitive ability. Intelligence, 35(1), 83-92. doi:10.1016/j.intell.2006.05.004 
Estudios Pedagógicos XLIII, N 1: 171-191, 2017 TALENTO ACADÉMICO, VULNERABILIDAD ESCOLAR Y RESULTADOS EN LA PRUEBA DE SELECCIÓN UNIVERSITARIA

Wolkowitz, A. (2008). A comparison of classical test theory \& item response theory methods for equating number-right scored to formula scored assessments. Ann Arbor: Pro Quest.

Zwick, R. (2002). Fair game?: The use of standardized admission tests in higher education. New York: Routledge Falmer. 
